\title{
Comparative Evaluation of Three Palliative Radiotherapy Schedules in Locally Advanced Head and Neck Cancer
}

\author{
Abhishek Soni ${ }^{\mathrm{a}, \mathrm{d}}$, Vivek Kaushal ${ }^{\mathrm{b}}$, Monica Verma ${ }^{\mathrm{c}}$, Anil Kumar Dhull ${ }^{\mathrm{b}}$, \\ Rajeev Atri ${ }^{b}$, Rakesh Dhankhar ${ }^{\mathrm{b}}$
}

\begin{abstract}
Background: The aim of the study was to evaluate and compare the efficacy, tolerability and toxicity of three palliative radiotherapy (RT) schedules in locally advanced head and neck carcinoma (LAHNC), i.e. Quad Shot schedule, Christie schedule and conventional palliative schedule.
\end{abstract}

Methods: The patients were randomly divided into three groups of 30 each. Group I patients were planned for 14.8 Gy in 4 fractions over 2 days, repeated three weekly for two more cycles. Group II patients were planned for 50 Gy in 16 fractions over 3.1 weeks. Group III patients were planned for 20 Gy in 5 fractions over 5 days, repeated after an interval of 3 weeks. The quality of life was assessed before and after RT using University of Washington Quality of Life questionnaire version 4 .

Results: Local control rates were $84 \%, 76 \%$, and $76 \%$ for groups I, II and III, respectively. Disease status at 6-month follow-up was no evidence of disease $(20 \%, 28 \%$, and $16 \%)$, residual disease $(72 \%$, $48 \%$, and $76 \%$ ), and recurrent disease $(8 \%, 24 \%$, and $8 \%$ ) in groups I, II and III, respectively. Grade III acute skin reactions were $28 \%, 44 \%$, and $16 \%$ in groups I, II and III, respectively. Grade III acute mucosal reactions were $36 \%, 56 \%$, and $24 \%$ in group I, II and III, respectively. Quality of life improved in all groups after RT.

Conclusion: Quad Shot schedule may be used in LAHNC with better local control and acceptable toxicity as compared to conventional palliative RT schedule in Indian setting.

Keywords: Christie; Head and neck carcinoma; Quad Shot; Quality

Manuscript accepted for publication January 19, 2017

aDepartment of Radiation Oncology, Indraprastha Apollo Hospital, Delhi, India

bDepartment of Radiotherapy, Pt. B.D. Sharma PGIMS, Rohtak, Haryana, India

'Department of Biochemistry, Pt. B.D. Sharma PGIMS, Rohtak, Haryana, India

${ }^{\mathrm{d} C o r r e s p o n d i n g ~ A u t h o r: ~ A b h i s h e k ~ S o n i, ~ D e p a r t m e n t ~ o f ~ R a d i a t i o n ~ O n c o l o g y, ~}$ Indraprastha Apollo Hospital, Delhi, India.

Email: abhisheksoni246@gmail.com

doi: https://doi.org/10.14740/wjon992w of life; Radiotherapy

\section{Introduction}

Head and neck cancer accounts for $4.8 \%$ of all cancers globally and $14.3 \%$ of all cancers in India [1]. In Indian setting, more than $70 \%$ of patients present in locally advanced stage and thus, local failure rates are as high as $50-70 \%[2,3]$. Radiotherapy (RT) alone is the standard non-surgical therapy for locally advanced head and neck carcinoma (LAHNC). For LAHNC, even the most effective RT regimens result in local control rates of $50-70 \%$ and disease-free survivals of $30-40 \%[4,5]$. Because of advanced disease at presentation, some of the patients are suitable only for palliative RT. The goal of treatment in these patients is to achieve prompt relief of distressing symptoms and one of the important factors that determine the treatment outcome is the quality of life (QOL). Since most of these patients have a poor performance status, prolonged duration of treatment and frequent visits to hospital compromise their QOL. Since improvement in symptoms along with QOL is an important aspect of palliation, a study without it is not worth. There have been some reports on the use of hypofractionated RT in the palliation of LAHNC but many are methodologically flawed, with limited or no toxicity data, and no assessment of QOL. There is no standard dose fractionation schedule of RT for palliative management of LAHNC. The present prospective, randomized study was planned to comparatively evaluate the efficacy, tolerability and toxicity of three schedules of palliative RT in LAHNC and assess QOL before and after RT in all groups using University of Washington Quality of Life (UWQOL) questionnaire version 4.

\section{Methods}

\section{Patient selection}

The present randomized, open label, parallel study was conducted on 90 treatment-naive, histopathologically proven patients of LAHNC. Patients receiving palliative RT for LAH- 
Table 1. Patient Characteristics

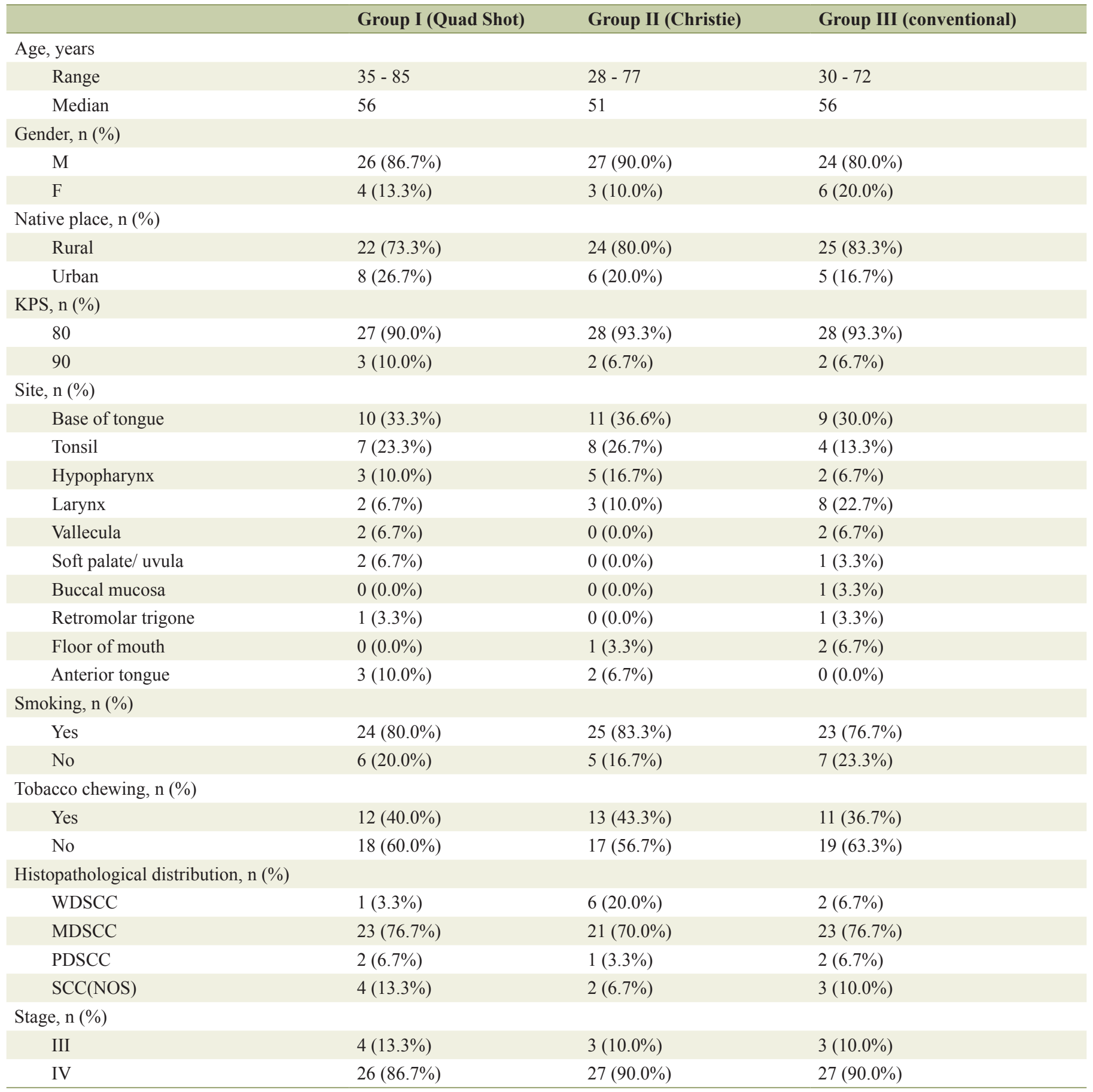

NC from April 2011 to June 2012 were randomly divided by simple random sampling in three groups of 30 patients each. The study was carried out after the approval of the protocol by the institution's review board. An informed consent was taken from all the patients and they were free to withdraw from the study at any point of time.

The inclusion criteria for the patients selected for the study were: Karnofsky performance status $(\mathrm{KPS}) \geq 70$, complete hemogram with $\mathrm{Hb}>8 \mathrm{~g} / \mathrm{dL}, \mathrm{TLC}>4,000 / \mathrm{mm}^{3}$, platelet count $>100,000 / \mathrm{mm}^{3}$, renal function tests with blood urea $<40 \mathrm{mg} /$ $\mathrm{dL}$ and serum creatinine $<1.5 \mathrm{mg} / \mathrm{dL}$, liver function tests with $\mathrm{SGOT} / \mathrm{PT}<35 / 40 \mathrm{IU} / \mathrm{L}$, AJCC stage III/IV and a positive biopsy for squamous cell carcinoma of head and neck. The patients having distant metastases, prior radiation, surgery or chemotherapy, KPS $<70$, pregnant or lactating patients, histopathology other than squamous cell carcinoma were excluded 
Table 2. Assessment of Quality of Life in Locally Advanced Head and Neck University of Washington Quality of Life Questionnaire Version $4(n=90)$

\begin{tabular}{|c|c|c|c|c|c|c|c|c|c|c|}
\hline \multirow{3}{*}{$\begin{array}{l}\text { Sr. } \\
\text { No. }\end{array}$} & \multirow{3}{*}{ Symptom } & \multicolumn{3}{|c|}{ Group I (Quad Shot) } & \multicolumn{3}{|c|}{ Group II (Christie) } & \multicolumn{3}{|c|}{ Group III (conventional) } \\
\hline & & \multicolumn{2}{|c|}{$\begin{array}{l}\text { Mean } \pm \text { standard } \\
\text { deviation }\end{array}$} & \multirow{2}{*}{$\begin{array}{l}P \\
\text { value }\end{array}$} & \multicolumn{2}{|c|}{$\begin{array}{l}\text { Mean } \pm \text { standard } \\
\text { deviation }\end{array}$} & \multirow{2}{*}{$\begin{array}{l}P \\
\text { value }\end{array}$} & \multicolumn{2}{|c|}{$\begin{array}{l}\text { Mean } \pm \text { standard } \\
\text { deviation }\end{array}$} & \multirow{2}{*}{$\begin{array}{l}P \\
\text { value }\end{array}$} \\
\hline & & Pre-RT & Post-RT & & Pre-RT & Post-RT & & Pre-RT & Post-RT & \\
\hline 1 & Pain & $50 \pm 15.54$ & $100 \pm 26.59$ & $0.001 *$ & $50 \pm 9.48$ & $75 \pm 29.16$ & $0.002 *$ & $50 \pm 13.02$ & $75 \pm 26.21$ & $0.001 *$ \\
\hline 2 & Appearance & $50 \pm 13.02$ & $75 \pm 16.08$ & $0.000 *$ & $50 \pm 8.64$ & $75 \pm 17.47$ & $0.000 *$ & $50 \pm 11.37$ & $75 \pm 15.99$ & $0.000^{*}$ \\
\hline 5 & Swallowing & $33 \pm 28.07$ & $67 \pm 27.58$ & 0.076 & $33 \pm 18.08$ & $67 \pm 30.12$ & $0.007^{*}$ & $33 \pm 22.70$ & $67 \pm 27.45$ & $0.021 *$ \\
\hline 6 & Chewing & $100 \pm 28.57$ & $75 \pm 25.43$ & 0.763 & $50 \pm 24.51$ & $50 \pm 24.91$ & 0.782 & $100 \pm 28.57$ & $75 \pm 25.43$ & 0.782 \\
\hline 7 & Speech & $83.5 \pm 26.75$ & $100 \pm 25.55$ & 0.968 & $67 \pm 16.17$ & $67 \pm 18.67$ & 0.705 & $100 \pm 28.30$ & $100 \pm 22.62$ & 0.713 \\
\hline 8 & Shoulder & $100 \pm 23.1$ & $100 \pm 16.99$ & 0.414 & $100 \pm 12.23$ & $100 \pm 12.23$ & 1 & $100 \pm 23.51$ & $100 \pm 17.00$ & 0.366 \\
\hline 12 & Anxiety & $33 \pm 21.11$ & $67 \pm 25.39$ & $0.005^{*}$ & $33 \pm 19.05$ & $67 \pm 23.92$ & 0.216 & $33 \pm 20.95$ & $67 \pm 22.21$ & $0.022 *$ \\
\hline 13 & Physical domain score & $50 \pm 13.22$ & $67 \pm 14.63$ & $0.043^{*}$ & $50 \pm 8.83$ & $61.33 \pm 13.63$ & 0.06 & $50 \pm 13.91$ & $67 \pm 12.52$ & $0.002 *$ \\
\hline 14 & Social domain score & $25 \pm 10.17$ & $75 \pm 31.95$ & $0.000^{*}$ & $25 \pm 0.00$ & $62.5 \pm 23.38$ & $0.000^{*}$ & $25 \pm 8.64$ & $75 \pm 7.45$ & $0.000 *$ \\
\hline 15 & HRQOL 7 days & $20 \pm 10.51$ & $60 \pm 22.38$ & $0.000^{*}$ & $20 \pm 3.65$ & $40 \pm 24.16$ & $0.000^{*}$ & $20 \pm 7.43$ & $60 \pm 20.44$ & $0.000 *$ \\
\hline 16 & Overall QOL & $20 \pm 10.51$ & $60 \pm 23.77$ & $0.000 *$ & $20 \pm 3.65$ & $40 \pm 24.16$ & $0.000 *$ & $20 \pm 7.43$ & $60 \pm 21.87$ & $0.000 *$ \\
\hline
\end{tabular}

Mean \pm standard deviation score for each domain using Wilcoxan signed rank test. *Statistically significant.

from the study.

Group I (Quad Shot schedule) patients were planned for radiation dose of $14.8 \mathrm{~Gy}$ in 4 fractions over a period of 2 days with 2 fractions per day of $3.7 \mathrm{~Gy}$ each, $6 \mathrm{~h}$ apart for two consecutive days. The same schedule was repeated for two more cycles with an interval of 3 weeks. The total planned radiation dose in this group was $44.4 \mathrm{~Gy} / 12 \# / 6.2$ weeks. Replanning with spinal cord sparing was done for last two fractions. Group II (Christie schedule) patients were planned for radiation dose of $50 \mathrm{~Gy}$ in 16 fractions over a period of 3.1 weeks with 3.125 Gy per fraction. Replanning with spinal cord sparing was done after 11 fractions. Group III (conventional schedule) patients were planned for radiation dose of $20 \mathrm{~Gy}$ in 5 fractions over a period of 5 days. The same schedule was repeated after an interval of 3 weeks. The total planned radiation dose in this group was $40 \mathrm{~Gy} / 10 \# / 3.5$ weeks. Replanning was done with spinal cord sparing for last fraction. All the patients were treated in a supine position. Two-dimensional planning was performed with a pretreatment simulation to work out the field borders which covered the primary tumor, disease extension and neck nodes. The patients were treated by parallel opposing fields and the dose was prescribed to the mid plane at the central axis. RT was delivered by cobalt- 60 .

Radiation reactions were assessed by Radiation Therapy Oncology Group (RTOG) criteria [6]. Tumor response (both primary and nodal response) was assessed by WHO response criteria either clinically or if needed, radiologically [7]. From the commencement of treatment, all the patients were regular- ly assessed daily during treatment and weekly during planned gaps in treatment. Detailed clinical evaluations were done by thorough local examination of the patients and all the patients were followed up regularly on outpatient basis for a period of at least 6 months at 1-month interval. Overall survival was measured from the day of diagnosis.

UWQOL questionnaire version 4 was used to evaluate QOL. It consists of 12 single question domains, these having between three and six response options that are scaled evenly from 0 (worst) to 100 (best) according to the hierarchy of response. The domains are pain, appearance, activity, recreation, swallowing, chewing, speech, shoulder, taste, saliva, mood and anxiety. The physical domain score, social domain score, health-related QOL in last 7 days (HRQOL 7 days) and overall QOL were also computed $[8,9]$. UWQOL questionnaire was assessed by treating radiation oncologist. The questions were translated into the local language for easy understanding of patients. The QOL was assessed at the beginning of treatment and 1 month after completion of planned treatment. The results of the study regarding completion of intended treatment, any interruptions in treatment, toxicity, local control rates and disease status at last follow-up in all the groups were documented.

\section{Statistical analysis}

The data were assessed and analyzed to find out difference in all the groups with the help of SPSS software. Compari- 


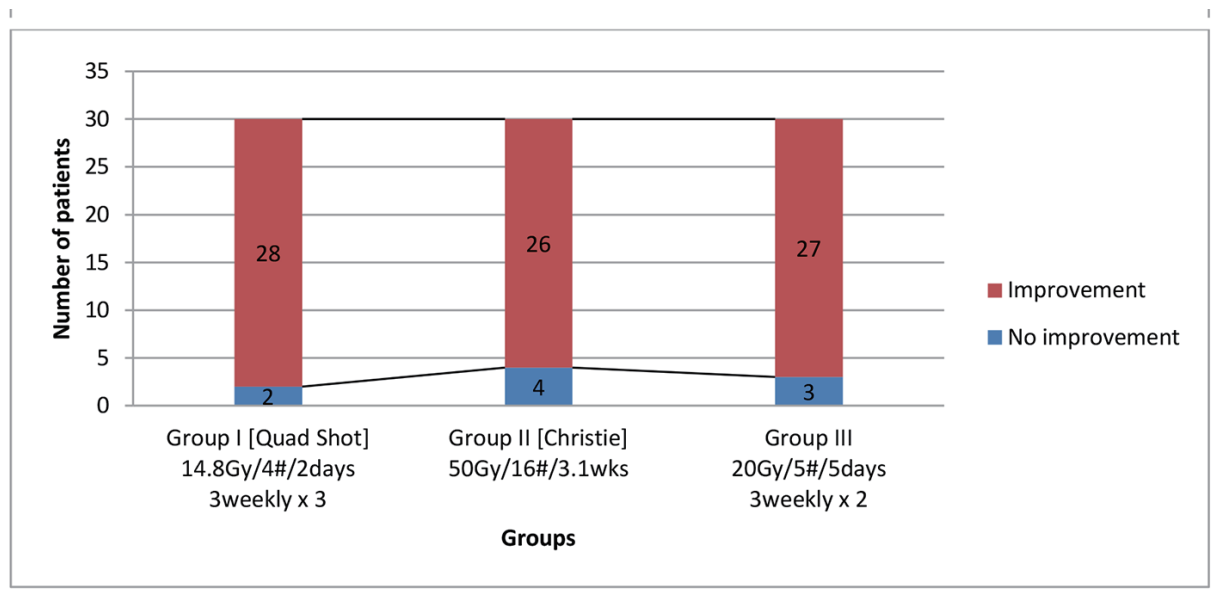

Figure 1. Locally advanced head and neck cancer comparison of three palliative radiotherapy schedules on overall quality of life $(\mathrm{n}=90)$. $\mathrm{P}$ values for group I $=0.4$, group II $=0.7$, group $\mathrm{III}=0.6$ (not significant) .

son between the groups for numeric variables was done using the Kruskal-Wallis test. Comparison between categorical measurements was done using the Chi-square test. Data were analyzed using IBM SPSS statistics 20. All P-values were twosided and P-values $<0.05$ were considered significant. Wilcoxan signed rank test was used for assessing the difference in QOL in three arms.

\section{Results}

The patient characteristics are shown in Table 1. The patient parameters were comparable in the three groups. The mean dose received by the patients was 43.41 Gy in group I, 47.29 Gy in group II and 40 Gy in group III. Twenty-eight patients in group I, 25 patients in group II and all patients in group III completed intended treatment. The dropout rate was $6.67 \%$ in group I, $16.67 \%$ in group II and nil in group III. Two patients in group I left the treatment after receiving two cycles of Quad Shot, due to grade 3 skin and mucosal reactions. Five patients in group II left the treatment after receiving 10, 11 and 12 fractions, respectively in two, two and one patients, due to grade 3 skin and mucosal reactions. These patients were not excluded from the study to minimize the bias. All the patients could complete the intended treatment in group III.

Using UWQOL questionnaire version 4, the mean \pm standard deviation scores of the individual domains, before and after radiation for group I, II and III, have been calculated and it is depicted in Table 2. The symptom status at presentation and after radiation treatment was documented in all the three groups. For group I, Quad Shot schedule, statistically significant improvements have been observed in scores of pain, appearance, activity, recreation, mood, anxiety, physical domain score, social domain score, HRQOL 7 days and overall QOL $(20 \pm 10.51$ to $60 \pm 23.77)$. Statistically significant decrement has been noted in taste and saliva score. For group II, Christie schedule, statistically significant improvements have been observed in scores of pain, appearance, activity, recreation, swallowing, mood, social domain, HRQOL 7 days and overall
QOL (20 \pm 3.65 to $40 \pm 24.16)$. Statistically highly significant decrement has been noted in taste and saliva score. For group III, conventional schedule, statistically significant improvements have been observed in scores of pain, appearance, activity, recreation, swallowing, mood, anxiety, physical domain, social domain, HRQOL 7 days and overall QOL $(20 \pm 7.43$ to $60 \pm 21.87)$. Statistically significant excrement has been noted in taste and saliva score. Although more improvement has been observed in group I, Quad Shot schedule, in physical domain score $(24,17$ and 22 patients in groups I, II and III, respectively), social domain score $(28,26$ and 27 patients in groups I, II and III, respectively), HRQOL 7 days $(28,25$ and 28 patients in groups I, II and III, respectively) and overall QOL (28, 26 and 27 patients in groups I, II and III, respectively), as compared to group II, Christie schedule, and group III, conventional schedule, but this was not statistically significant (using Chi-square test) and this is depicted in Figure 1.

Locoregional control after the completion of treatment was complete response (CR) $(30 \%, 43.33 \%$ and $26.67 \%)$, partial response (PR) $(53.33 \%, 36.67 \%$ and $50 \%)$, and no response (NR) $(16.67 \%, 20 \%$ and $23.33 \%)$ in groups I, II and III, respectively, but this was not statistically significant $(\mathrm{P}=$ 0.350 ) and Figure 2 shows the same. The patients were followed for a minimum period of 6 months (range 6 - 19 months, median follow-up of 11 months). Disease status at the end of 6 months was no evidence of disease (NED) $(20 \%, 26.67 \%$ and $13.33 \%)$, residual disease (RD) $(70 \%, 56.67 \%$ and $76.67 \%)$, and recurrent disease (REC) $(10 \%, 16.67 \%$ and $10 \%)$ in groups I, II and III respectively, but not statistically significant $(\mathrm{P}=0.627)$. Grade III radiation skin reactions were $23.33 \%$, $40 \%$ and $20 \%$ in groups I, II and III, respectively, not statistically significant $(\mathrm{P}=0.175)$. Grade III radiation mucosal reactions were $36.66 \%, 53.33 \%$ and $23.33 \%$, in groups I, II and III, respectively, statistically significant in group II, Christie schedule. Late mucosal toxicity was statistically significantly higher $(\mathrm{P}=0.002)$ in group II, Christie schedule, as compared to groups I and III. The difference in pattern of failure was not statistically significant among the three groups which are shown in Table 3. Failure included residual, persistent and re- 


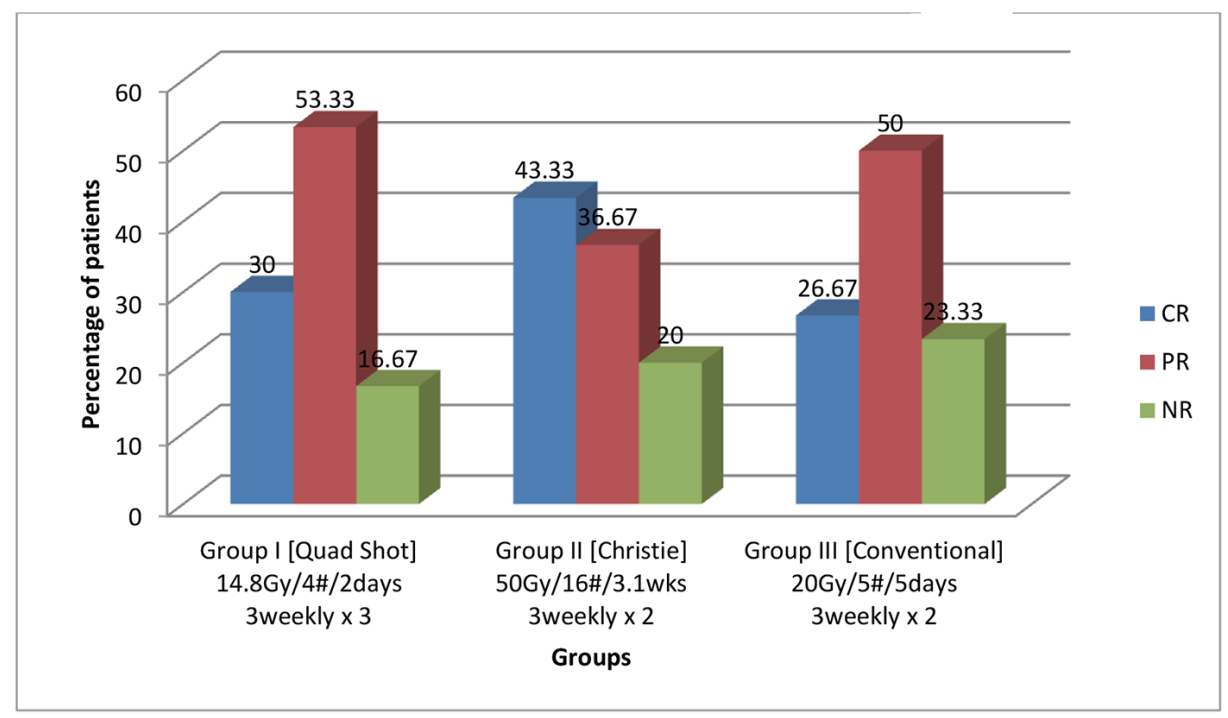

Figure 2. Locally advanced head and neck cancer comparison of three palliative radiotherapy schedules on locoregional control at the end of treatment (percentage). $\mathrm{P}$ values for overall response rate of group I $=0.5$, group II $=0.6$, group III $=0.3$ (not significant).

current disease. Primary site failure was seen in $66.67 \%, 70 \%$ and $72.22 \%$ patients of groups I, II and III, respectively. Nodal failure was seen in $60 \%, 60 \%$ and $58.89 \%$ patients of groups I, II and III, respectively. Both primary and nodal failures were seen in $46.67 \%, 56.67 \%$ and $52.22 \%$ patients of groups I, II and III, respectively. Response rates were $83.33 \%, 80 \%$ and $76.67 \%$ for groups I, II and III, respectively, not statistically significant $(\mathrm{P}=0.350)$. Median survival was 11.5 months in group I, 10.5 months in group II and 11 months in group III, not statistically significant. Overall survival rates at 1 year were $40 \%, 36.67 \%$ and $33.33 \%$ in group I, II and III, respectively and Figure 3 shows it.

\section{Discussion}

To the best of our knowledge, this is the first open label, prospective, randomized study comparing three palliative RT schedules for the management of LAHNC. In LAHNC, surgery without adjuvant RT is associated with very poor cure rates. Compared with surgery alone, adjuvant RT resulted in an approximately $10 \%$ absolute increase in 5-year cancer-specific survival and overall survival. Chemotherapy alone is also not curative and it is used mostly along with RT. So RT with or without chemotherapy remains the mainstay of treatment in LAHNC [10-12]. Because of advanced stage at the time of presentation, the local failure rates are as high as $50-70 \%$, despite improvement in treatment strategies for the management of LAHNC [13]. There is no standard dose fractionation schedule of RT for palliative management of LAHNC. Different authors have followed different schedules of total dose and numbers of fractions of RT for achieving palliation in LAHNC.

The cutaneous radiation reactions follow a definite pattern following conventional RT. In our study, the skin changes were consistent with those described in literature [6]. Hypofraction-

Table 3. Pattern of Failure at Last Follow-Up

\begin{tabular}{llllll}
\hline Group & Stage & NED & Primary site failure & Nodal failure & Both primary and nodal failure \\
\hline Group I Quad shot & III & $0 / 4(0.00 \%)$ & $3 / 4(75.00 \%)$ & $2 / 4(50.00 \%)$ & $1 / 4(25.00 \%)$ \\
& IV & $6 / 26(23.07 \%)$ & $17 / 26(65.38 \%)$ & $16 / 26(61.54 \%)$ & $13 / 26(50.00 \%)$ \\
& Total & $6 / 30(2.00 \%)$ & $20 / 30(66.67 \%)$ & $18 / 30(60.00 \%)$ & $14 / 30(46.67 \%)$ \\
Group II Christie & III & $2 / 3(66.67 \%)$ & $1 / 3(33.33 \%)$ & $0 / 3(0.00 \%)$ & $0 / 3(0.00 \%)$ \\
& IV & $6 / 27(22.22 \%)$ & $20 / 27(74.07 \%)$ & $18 / 27(66.67 \%)$ & $17 / 27(62.96 \%)$ \\
& Total & $8 / 30(26.67 \%)$ & $21 / 30(70.00 \%)$ & $18 / 30(60.00 \%)$ & $17 / 30(56.67 \%)$ \\
Group III conventional & III & $0 / 3(0.00 \%)$ & $3 / 3(100.00 \%)$ & $2 / 3(66.67 \%)$ & $2 / 3(66.67 \%)$ \\
& IV & $4 / 27(14.81 \%)$ & $21 / 27(77.77 \%)$ & $15 / 27(55.55 \%)$ & $14 / 27(51.85 \%)$ \\
& Total & $4 / 30(13.33 \%)$ & $24 / 30(80.00 \%)$ & $17 / 30(56.67 \%)$ & $16 / 30(53.33 \%)$ \\
\hline
\end{tabular}




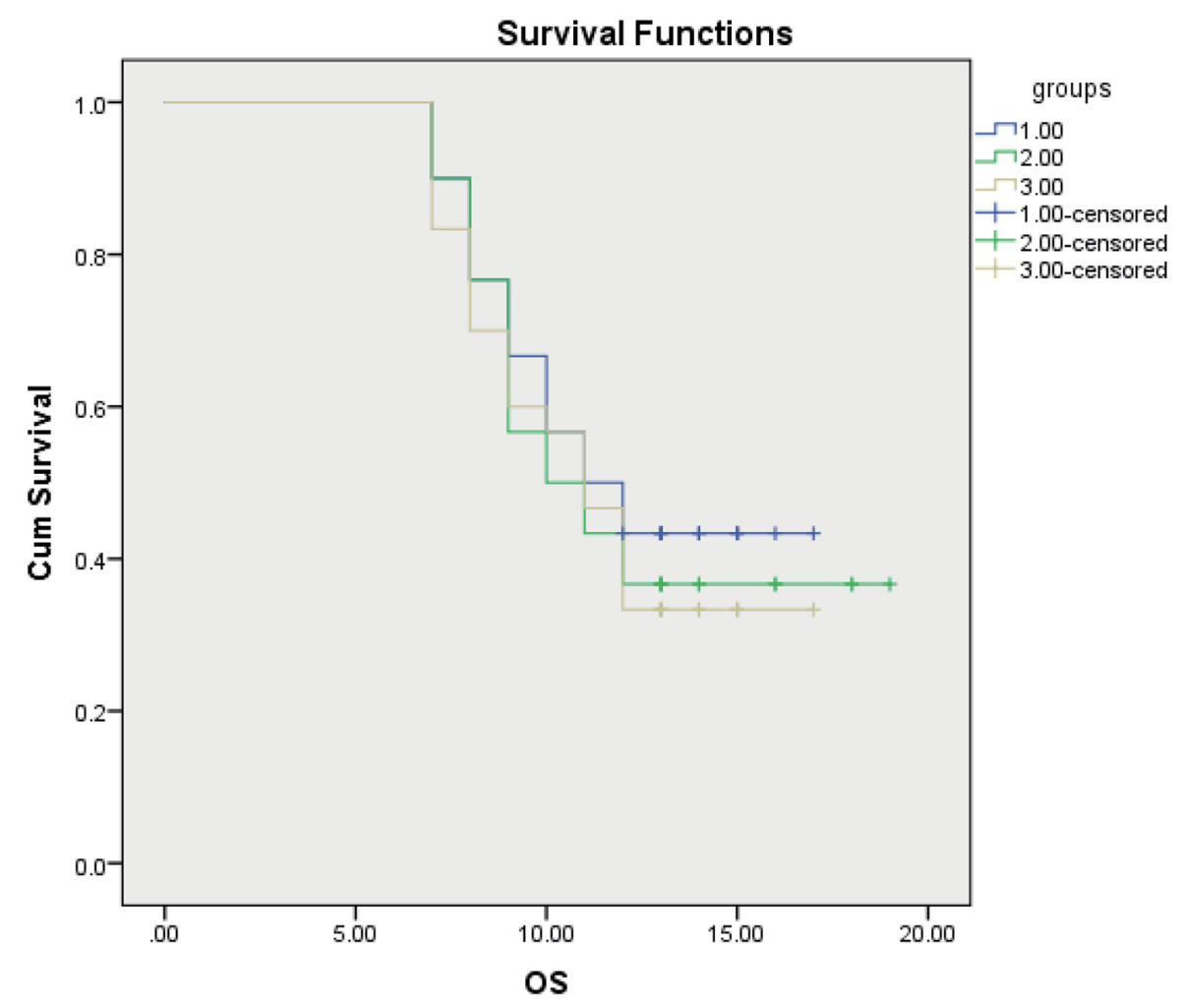

\begin{tabular}{|l|c|c|}
\hline GROUP & $\begin{array}{l}\text { OVERALL SURVIVAL } \\
\text { AT 1 YEAR }\end{array}$ & P value \\
\hline $\begin{array}{l}\text { GROUP I } \\
\text { QUAD SHOT SCHEDULE }\end{array}$ & $\mathbf{4 0 . 0 0 \%}$ & 0.5 (NS) \\
\hline $\begin{array}{l}\text { GROUP II } \\
\text { CHRISTIE SCHEDULE }\end{array}$ & $36.67 \%$ & 0.3 (NS) \\
\hline $\begin{array}{l}\text { GROUP III } \\
\text { CONVENTIONAL SCHEDULE }\end{array}$ & $33.33 \%$ & 0.5 (NS) \\
\hline
\end{tabular}

Figure 3. Kaplan-Meir survival curve showing overall survival for all the groups (in months along the x-axis).

ation has been known to produce greater overall toxicity in head and neck cancer patients. Similar trend was seen in our study. The difference in the three groups was statistically not significant. In the present study, grade 2 acute skin reaction in group I was seen in $>50 \%$ of the patients, and grade 3 skin reaction was seen in around $20 \%$ of the patients which is in accordance to the study by Chen et al [14]. In group II, Christie group, grade 2 and 3 acute skin toxicity was seen in $60 \%$ and $40 \%$ of the patients, which is in accordance to the study by AlMamgani et al [15]. In group III, grade 2 and 3 acute skin toxicity was seen in $>50 \%$ and $20 \%$ of the patients, respectively, which is similar to study by Mohanti et al [16]. Another hypofractionated trial for similar patients by Porceddu et al reported $26 \%$ grade 3 mucositis and $11 \%$ grade 3 skin toxicity, which supports the toxicity data in the present study [17].

The severity of radiation-induced mucositis depends on the total dose, fractionation and duration of therapy and associated infections [6]. Grade 3 acute mucosal reactions in group I was seen in approximately $30 \%$ of the patients, which is slightly higher as compared to study by Chen et al [14], which may be due to poor oral intake, poor hygiene or nonaffordability for the medicines in the Indian setting. Grade 3 acute mucosal toxicity in group II was seen in $>50 \%$ of the patients, which is similar to study by Al-Mamgani et al [15]. Grade 2 acute mucosal toxicity ( $>50 \%$ ) in group III is in accordance to Mohanti et al [16]. By the end of the treatment grade 3 mucosal toxicity was statistically significantly $(\mathrm{P}<0.05)$ higher in group II, Christie group. RTOG grade 4 reactions were not seen in any of the patients. None of the patients in any group developed grade 4 hematological toxicity. Hematological and TLC toxicity were comparable to that reported by Chen et al [14].

In group I, Quad Shot, response rate at the end of the treatment was $83.33 \%$ with complete response in $30 \%$ of patients (including complete primary tumor response in $36.66 \%$ and complete nodal response in $46.6 \%$ ), which is comparable to Chen et al [14] and Corry et al [18]. Our results are comparable to Carrascosa et al [19] and Paris et al [20] in providing good tumor response and palliation of symptoms [19]. In the present study in group II, Christie group, complete response 
was seen in $43.3 \%$, partial response in $36.7 \%$, with an overall response rate of $80 \%$, and rest $20 \%$ patients are non-responsive. The data closely match to study by Al-Mamgani et al [15] and Chen et al [14]. In group III, response rate at the end of treatment was $76.7 \%$, with a complete response rate of $26.7 \%$, which is in vicinity of Chen et al [14] and Agarwal et al [21]. Acute and late reactions were acceptable [21]. Late radiation toxicity was observed as grade 3 cutaneous and mucosal in $3.3 \%$ in group I, $6.7 \%$ and $10 \%$ respectively in group II, which is in accordance with that reported by Corry et al [18], Ghoshal et al [17] for group I and Al-Mamgani et al [15] for group II, respectively.

\section{Quality of life}

The aim of palliative radiation is to relieve the symptoms quickly while minimizing the side effects. In addition, the treatment should be delivered in the shortest possible time considering patients' and caregivers' convenience. Ghoshal et al evaluated symptom relief and QOL in LAHNC using Quad Shot schedule [22]. UWQOL questionnaire was used for assessing QOL before and after radiation. After the first course, all patients had good symptom relief, improvement in QOL, and $86.7 \%$ patients had more than $50 \%$ objective response [22]. In another study by Ghoshal et al, at 1-month post-treatment, $100 \%$ patients with pain and $>90 \%$ patients with dysphagia, dyspnea and insomnia experienced $>50 \%$ symptom relief. Cough was relieved in $60 \%$ patients [23]. Mohanti et al treated patients with stage 4 LAHNC with a uniform dose of $20 \mathrm{~Gy} / 5$ fractions over 1 week. Good symptom relief $(50 \%$ or more) was found in pain, dysphagia, hoarseness, otalgia, respiratory distress and cough [16]. Corry et al tried Quad Shot, which yielded $53 \%$ objective response. In $67 \%$ patients, the performance status stabilized or improved. The treatment was well tolerated, with overall improved QOL in 44\% patients. Treatment was felt to be worthwhile by $43 \%, 58 \%$ and $63 \%$ of patients after first, second and third courses of cyclical radiation, respectively [18]. Al-Mamgani et al, by using Christie schedule, achieved excellent palliation resulting in acceptable response rates, excellent symptom control, acceptable toxicity profile and good QOL of patients [15]. Results from these studies are comparable to the present study. The present study shows statistically significant improvements in the physical domain score, social domain score, HRQOL 7 days and overall QOL in all the groups but these improvements are slightly higher in group I, Quad Shot group as compared to groups II and III.

\section{Limitations and bias}

There are certain limitations of the study. Larger number of patients is required for a more validated result of the study. Although patients of poor socio-economic status were included in the study, some patients were not economically strong enough to purchase medicines meant for palliative management and this might have confounded the results of QOL among the groups.

\section{Conclusion}

The aim of palliative radiation in any advanced cancer is to relieve the symptoms quickly while minimizing the side effects. In addition, the treatment should be delivered in the shortest possible time considering patients' and caregivers' convenience. To conclude, group III, conventional palliative RT schedule was tolerated by all the patients without significant problems; however, it yielded the least local control out of the three groups. Christie schedule (group II) showed better complete response than any other palliative schedule but was associated with dropout rate of $16.67 \%$ because of severe radiation reactions. The Quad Shot schedule showed optimum local control and radiation reactions amongst three groups. The present study shows a statistically significant improvement in most of the domains in all three groups but these improvements were slightly higher in Quad Shot group as compared to groups II and III. Taking into account the comparable response rate, comparable symptom relief, slightly more improvement in the post-treatment QOL, significant less toxicities than group II and least frequent hospital visits for the treatment as compared to groups II and III, group I, Quad Shot regimen may be recommended in the palliative setting in LAHNC.

\section{Acknowledgments}

There was no source of financial grant or other funding. The contribution of colleagues or institutions is highly acknowledged.

\section{Conflicts of Interest}

The authors declare that they have no conflicts of interest.

\section{References}

1. Ferlay J, Soerjomataram I, Ervik M, et al. GLOBOCAN 2012 v1.0. Cancer Incidence and Mortality. 2013.

2. Lamont EB, Vokes EE. Chemotherapy in the management of squamous-cell carcinoma of the head and neck. Lancet Oncol. 2001;2(5):261-269.

3. Stupp R, Weichselbaum RR, Vokes EE. Combined modality therapy of head and neck cancer. Semin Oncol. 1994;21(3):349-358.

4. Overgaard J, Hansen HS, Specht L, Overgaard M, Grau C, Andersen E, Bentzen J, et al. Five compared with six fractions per week of conventional radiotherapy of squamous-cell carcinoma of head and neck: DAHANCA 6 and 7 randomised controlled trial. Lancet. 2003;362(9388):933-940.

5. Fein DA, Lee WR, Amos WR, Hinerman RW, Parsons JT, Mendenhall WM, Stringer SP, et al. Oropharyngeal carcinoma treated with radiotherapy: a 30-year experience. Int J Radiat Oncol Biol Phys. 1996;34(2):289-296. 
6. Cox JD, Stetz J, Pajak TF. Toxicity criteria of the Radiation Therapy Oncology Group (RTOG) and the European Organization for Research and Treatment of Cancer (EORTC). Int J Radiat Oncol Biol Phys. 1995;31(5):13411346.

7. De Jager R, Longeval E, Klastersky J. High-dose cisplatin with fluid and mannitol-induced diuresis in advanced lung cancer: a phase II clinical trial of the EORTC Lung Cancer Working Party (Belgium). Cancer Treat Rep. 1980;64(12):1341-1346.

8. Hassan SJ, Weymuller EA, Jr. Assessment of quality of life in head and neck cancer patients. Head Neck. 1993;15(6):485-496.

9. Rogers SN, Gwanne S, Lowe D, Humphris G, Yueh B, Weymuller EA, Jr. The addition of mood and anxiety domains to the University of Washington quality of life scale. Head Neck. 2002;24(6):521-529.

10. Lavaf A, Genden EM, Cesaretti JA, Packer S, Kao J. Adjuvant radiotherapy improves overall survival for patients with lymph node-positive head and neck squamous cell carcinoma. Cancer. 2008;112(3):535-543.

11. Mendenhall WM, Morris CG, Amdur RJ, Hinerman RW, Malyapa RS, Werning JW, Lansford CD, et al. Definitive radiotherapy for tonsillar squamous cell carcinoma. Am J Clin Oncol. 2006;29(3):290-297.

12. Perez CA, Carmichael T, Devineni VR, Simpson JR, Frederickson J, Sessions D, Spector G, et al. Carcinoma of the tonsillar fossa: a nonrandomized comparison of irradiation alone or combined with surgery: long-term results. Head Neck. 1991;13(4):282-290.

13. Brizel DM, Adelstein DJ. Locally Advanced Squamous Cell Carcinoma of Head and Neck. In: Halperin EC, Wazer DE, Perez CA, Brady LW (eds). Principles and Practice of Radiation Oncology, 6th ed. Philadelphia: Lippincott Williams and Wilkins, 2013; p. 718-730.

14. Chen AM, Vaughan A, Narayan S, Vijayakumar S. Palliative radiation therapy for head and neck cancer: toward an optimal fractionation scheme. Head Neck. 2008;30(12):1586-1591.
15. Al-mamgani A, Tans L, Van rooij PH, Noever I, Baatenburg de jong RJ, Levendag PC. Hypofractionated radiotherapy denoted as the "Christie scheme": an effective means of palliating patients with head and neck cancers not suitable for curative treatment. Acta Oncol. 2009;48(4):562-570.

16. Mohanti BK, Umapathy H, Bahadur S, Thakar A, Pathy S. Short course palliative radiotherapy of 20 Gy in 5 fractions for advanced and incurable head and neck cancer: AIIMS study. Radiother Oncol. 2004;71(3):275-280.

17. Porceddu SV, Rosser B, Burmeister BH, Jones M, Hickey B, Baumann K, Gogna K, et al. Hypofractionated radiotherapy for the palliation of advanced head and neck cancer in patients unsuitable for curative treatment - "Hypo Trial". Radiother Oncol. 2007;85(3):456-462.

18. Corry J, Peters LJ, Costa ID, Milner AD, Fawns H, Rischin D, Porceddu S. The 'QUAD SHOT' - a phase II study of palliative radiotherapy for incurable head and neck cancer. Radiother Oncol. 2005;77(2):137-142.

19. Carrascosa LA, Yashar CM, Paris KJ, Larocca RV, Faught SR, Spanos WJ. Palliation of pelvic and head and neck cancer with paclitaxel and a novel radiotherapy regimen. J Palliat Med. 2007;10(4):877-881.

20. Paris KJ, Spanos WJ, Jr., Lindberg RD, Jose B, Albrink F. Phase I-II study of multiple daily fractions for palliation of advanced head and neck malignancies. Int J Radiat Oncol Biol Phys. 1993;25(4):657-660.

21. Agarwal JP, Nemade B, Murthy V, Ghosh-Laskar S, Budrukkar A, Gupta T, D'Cruz A, et al. Hypofractionated, palliative radiotherapy for advanced head and neck cancer. Radiother Oncol. 2008;89(1):51-56.

22. Ghoshal S, Chakraborty S, Moudgil N, Kaur M, Patel FD. Quad shot: a short but effective schedule for palliative radiation for head and neck carcinoma. Indian $\mathrm{J}$ Palliat Care. 2009; 15(2):137-140.

23. Ghoshal S, Patel F, Mudgil N, Bansal M, Sharma S. Palliative radiotherapy in locally advanced head and neck cancer: A prospective trial. Indian J Palliat Care. 2004;10:1923. 